\title{
Penicillium chrysogenum as a model system for studying cellular effects of methylglyoxal
}

\author{
Christian Q. Scheckhuber
}

\begin{abstract}
Background: a-oxoaldehydes are formed as toxic by-products during metabolic activity. The biologically most important compound of this class, methylglyoxal, results from spontaneous phosphate elimination from dihydroxyacetone phosphate and glyceraldehyde 3-phosphate which are intermediate glycolysis products. Methylglyoxal-mediated modification of lipids, nucleic acids and proteins is known to lead to the formation of advanced glycation end products. These modifications contribute to the aetiology of severe diseases like diabetes and neurodegenerative disorders. By using simple model organisms it is possible to conveniently study the effects of methylglyoxal on cellular processes. Here, results are presented on the effects of methylglyoxal on mycelium growth, stationary phase entry (monitored by autophagy induction), mitochondrial morphology and protein composition in the filamentous fungus Penicillium chrysogenum.

Results: Methylglyoxal leads to growth rate reduction of this fungus so that the entry into the stationary phase is delayed. Mitochondrial morphology is not changed by methylglyoxal. However, rapamycin-mediated fragmentation of mitochondria is prevented by methylglyoxal. Furthermore, three proteins are identified that are present in lower abundance when methylglyoxal is added to the growth medium (aldo-keto reductase [Pc22g04850], 5-methyl-tetrahydropteroyl-triglutamate-homocysteine S-methyltransferase [Pc22g18630] and NAD-dependent formate dehydrogenase [Pc12g04310]).
\end{abstract}

Conclusions: The presented results contribute to the understanding of cellular pathways and mechanisms that are affected by the ubiquitous a-oxoaldehyde methylglyoxal.

Keywords: Autophagy, Green-fluorescent protein, Methylglyoxal, Mitochondria, Penicillium chrysogenum, Peroxisomes, Stationary phase

\section{Background}

Living cells depend on the enzymatic conversion of Dglucose to pyruvate via glycolysis for the formation of the molecular energy stores adenosine triphosphate (ATP) and nicotinamide adenine dinucleotide (NADH). Glycolysis, however, can also lead to the production of toxic byproducts. The best studied and probably the most relevant compound of this class is the $\alpha$-oxoaldehyde methylglyoxal (also known as pyruvaldehyde or 2-oxopropanal) [1]. Methylglyoxal is mainly formed by spontaneous elimination of phosphate from the glycolysis intermediates dihydroxyacetone phosphate (DHAP) and, to a lesser extent, glyceraldehyde 3-phosphate [2]. Another metabolic route

Correspondence: Christian.Scheckhuber@senckenberg.de Senckenberg Research Institute, LOEWE Excellence Cluster for Integrative Fungal Research (IPF), Georg-Voigt-Str. 14-16, D-60325 Frankfurt am Main, Germany that leads to the formation of methylglyoxal is the oxidation of aminoacetone in the catabolism of L-threonine which is mediated by the semicarbazide-sensitive amine oxidase [3]. Methylglyoxal can also be formed as a sideproduct of lipid peroxidation under conditions of elevated intracellular oxidative stress [4]. This $\alpha$-oxoaldehyde causes protein glycation and functional impairments due to its high reactivity with amino groups of proteinaceous lysine and arginine residues [5]. These residues are modified further and ultimately converted into 'advanced glycation end-products' (AGEs). For example, a major type of AGE in cells of the human eye lens from aged donors is the hydroimidazolone $\mathrm{N}^{\varepsilon}$-(5-hydro-5-methyl-4-imidazolon-2-yl)-ornithine (MG-H1) [6]. Importantly, the MG-H1 modification is found to be also increased in pathological conditions like Alzheimer's disease, arthritis, diabetes, renal failure and Parkinson's disease [7]. Collagen type IV 
$[8]$ and hemoglobin $[9,10]$ in humans and enolase 2 in baker's yeast [11] have been identified as main targets of methylglyoxal-mediated modifications. Collagen type IV loses its ability to interact with integrins whereas the function of haemoglobin is compromised by an intensified oxygen binding capacity. For yeast enolase 2 studies have been performed in which in vivo and in vitro glycation patterns after methylglyoxal treatment were compared. It was revealed that in vivo glycation of enolase 2 is a specific process where certain lysine and arginine amino acids are consistently modified.

Unsurprisingly, cells possess protective mechanisms that prevent or limit glycation damage. The best characterised enzymatic system that detoxifies methylglyoxal by conversion to D-lactate consists of three components: glyoxalase I (GLO1, lactoylglutathione lyase), glyoxalase II (GLO2, hydroxyacylglutathione hydrolase) and catalytic amounts of reduced glutathione [12]. One example demonstrating the importance of the glyoxalase system for protecting organisms from the deleterious effects of $\alpha$-oxoaldehydes and subsequent glycation stems from work on nematodes. Overexpression of the Glo1 gene in Caenorhabditis elegans was found to increase the lifespan of this organism by protecting components of the mitochondrial respiratory chain from glycation [13]. In addition to glyoxalase I and glyoxalase II a novel type of glyoxalase has been reported that is working independently of glutathione [14]. Mutant variants of this enzyme (DJ-1/PARK7) have been reported to contribute to the aetiology of autosomal recessive early-onset Parkinsonism [15]. Later studies revealed that some of these mutations affect mitochondrial integrity in cellular models of Parkinsonism [16, 17]. Recently, it was demonstrated that D-lactate, which is also formed by DJ-1, is able to positively influence mitochondrial function by maintaining the membrane potential of these organelles [18]. Collectively these results demonstrate that not only the removal of methylglyoxal but also an end-product of glyoxalase activity is important for maintaining cellular health and integrity. Other enzymes that may limit the formation of methylglyoxal are methylglyoxal reductase [19], aldo-keto reductases [20], $\alpha$-oxoaldehyde dehydrogenase and betaine dehydrogenase [21] although their contribution to methylglyoal degradation has been investigated less.

Recently the role of methylglyoxal and its degradation by the glyoxalase system on fungal senescence was studied in the filamentous ascomycete Podospora anserina [22]. It was shown that overexpression of genes encoding glyoxalase I (PaGlo1) and glyoxalase II (PaGlo2) has a beneficial effect on viability and delayed the onset of senescence in this model system while the deletion of PaGlo1 resulted in a strongly decreased lifespan compared to the wild type when the strains were grown in the presence of $2 \%(\mathrm{w} / \mathrm{v})$ glucose which is supposed to increase the formation of methylglyoxal. These results suggest a link between intracellular methylglyoxal levels and fungal viability. Subsequently, the impact of increased glyoxalase I and II levels was studied in an industrial producer of $\beta$-lactam antibiotics, Penicillium chrysogenum [23]. Overexpression of PcGlo1 and PcGlo2 led to the improvement of penicillin (PEN) production in this fungus. Analysis of protein levels revealed that increased levels of two enzymes of the biosynthetic pathway for PEN are present in the PcGlo1/PcGlo2 overexpression strain. One of these enzymes, isopenicillin-N acyltransferase (IAT), is localised to peroxisomes [24], organelles of anti-oxidative activity and sites of various pathways of secondary metabolism.

The aim of the present study was to elucidate the effects of exogenous methylglyoxal on mycelium growth, initiation of the stationary phase, morphology of mitochondria and overall protein composition in P. chrysogenum. It is revealed that methylglyoxal affects the growth rate of the mycelium so that initiation of the stationary phase is delayed. Methylglyoxal has no effect on mitochondrial morphology: thread-like mitochondria are present in mycelia independent of methylglyoxal treatment. By using differential isoelectric focusing (IEF)/twodimensional sodium dodecyl sulphate polyacrylamide electrophoresis (2D-SDS-PAGE) in combination with peptide mass fingerprinting three proteins that are present in lower abundance compared to the control when methylglyoxal is added to the growth medium are identified.

\section{Results}

\section{Effects of methylglyoxal on mycelium growth}

Although the consequences of overexpression of the glyoxalase-encoding genes PcGlo1 and PcGlo2 for penicillin production have recently been studied [23] the effects of exogenously added methylglyoxal on the growth behaviour of $P$. chrysogenum have so far not been investigated. The P. chrysogenum strain Ws54-1255 (GFP-SKL) was grown on agarose pads without added nutrients, 'starvation pads' [25], in the presence or absence of exogenously added methylglyoxal and analysed by light microscopy (Fig. 1). A methylglyoxal concentration of $0.05 \%$ $(\mathrm{v} / \mathrm{v})$ was found to be suited for the studies because lower concentrations (e. g., $0.005 \%[\mathrm{v} / \mathrm{v}]$ and $0.01 \%[\mathrm{v} / \mathrm{v}]$ ) cause no observable changes on the growth behaviour and higher concentrations (e. g., $0.1 \%[\mathrm{v} / \mathrm{v}]$ ) led to almost complete inhibition of growth. The untreated control shows pronounced growth after $20 \mathrm{~h}$ and $40 \mathrm{~h}$ of incubation (Fig. 1a). At $60 \mathrm{~h}$ roughly the same amount of mycelium is present compared to $40 \mathrm{~h}$ which is an indication for a stop of cell growth. This is probably based on nutrient limitation encountered by the mycelium after prolonged growth on the starvation pads. The samples subjected to $0.05 \%(\mathrm{v} / \mathrm{v})$ methylglyoxal grow initially $(20 \mathrm{~h})$ much 

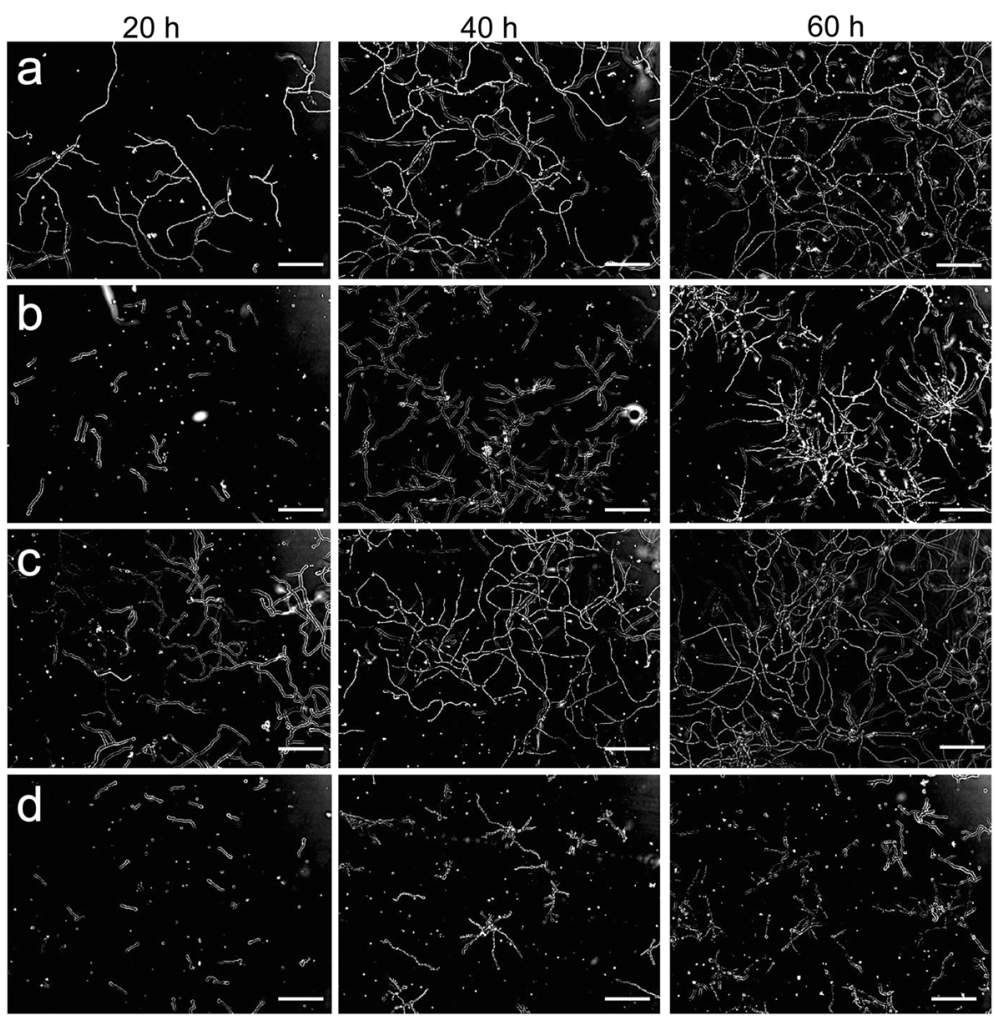

Fig. 1 Effects of methylglyoxal and rapamycin on growth of $P$. chrysogenum Ws54-1255 (GFP-SKL). At the indicated times pictures from cultures grown on starvation pads were taken at the indicated time points. Representative images are shown for each time point. a control; $\mathbf{b}+0.05 \%$ $(\mathrm{v} / \mathrm{v})$ methylglyoxal; $\mathbf{c}+1 \mu \mathrm{M}$ rapamycin; $\mathbf{d}+0.05 \%(\mathrm{v} / \mathrm{v})$ methylglyoxal/+ $1 \mu \mathrm{M}$ rapamycin. Scale bars: $100 \mu \mathrm{m}$

more slowly compared to the control (Fig. 1b). This retardation of growth demonstrates that methylglyoxal can enter the fungal cells and interfere with their function. At later time points $(40 \mathrm{~h}$ and $60 \mathrm{~h})$ the colonies are still growing although their morphology looks quite irregular (Fig. 1b). In samples treated with $1 \mu \mathrm{M}$ rapamycin for maximum induction of autophagy mycelium growth stalls (similar to the untreated control) at $40 \mathrm{~h}$ (Fig. 1c). Rapamycin is a well-known inhibitor of TOR ('target of rapamycin') signalling [26]. This treatment leads to pronounced induction of autophagy in most organisms [27]. When rapamycin and methylglyoxal are added in combination mycelium growth at all time points is reduced even further compared to samples subjected solely to methylglyoxal (Fig. 1d). Therefore rapamycin treatment seems to sensitize $P$. chrysogenum towards exogenously added methylglyoxal.

The observed differences in mycelium growth in the presence or absence of methylglyoxal could be accompanied by a delayed entry into stationary phase. In filamentous fungi, autophagy induction is a marker for stationary phase entry. Therefore degradation of GFP-SKL labelled peroxisomes as an autophagy marker was analysed by fluorescence microscopy (Fig. 2, Table 1). The utilised assay is based on the principle that GFP is not efficiently degraded by proteases localized in vacuoles [28]. Therefore the presence of GFP in vacuoles is a measure for peroxisomal degradation in the Ws54-1255 (GFP-SKL) reporter strain. In untreated samples vacuoles filled with GFP become visible after $40 \mathrm{~h}$ of incubation and these are also present after $60 \mathrm{~h}$ (Fig. 2a). At the later time point GFP localized to the vacuole lumen is observed in ca. $50 \%$ of hyphae (Table 1). By contrast, in samples subjected to $0.05 \%(\mathrm{v} / \mathrm{v})$ methylglyoxal no GFP-labelled vacuoles are observed up to $40 \mathrm{~h}$ (Fig. 2b, Table 1) indicating that these mycelia have not entered stationary phase by this time point. At a later time point $(60 \mathrm{~h})$ GFP-labelled vacuoles become visible (Fig. 2b) but only in approximately $25 \%$ of hyphae (Table 1 ). After $40 \mathrm{~h}$ pronounced vacuolation of hyphae is observed when $1 \mu \mathrm{M}$ rapamycin was added to the samples (Fig. 2c, Table 1). These vacuoles contain GFP suggesting peroxisomal degradation by autophagy (pexophagy). At $60 \mathrm{~h}$ almost all hyphae contain GFP-labelled vacuoles (Table 1) which are differing in morphology from vacuoles observed at $40 \mathrm{~h}$ (their structure seems less regular and they are filled with granulate matter which might be due to the high level of degradation occurring in strains that are subjected to both starvation and rapamycin). When rapamycin and methylglyoxal are synergistically applied 


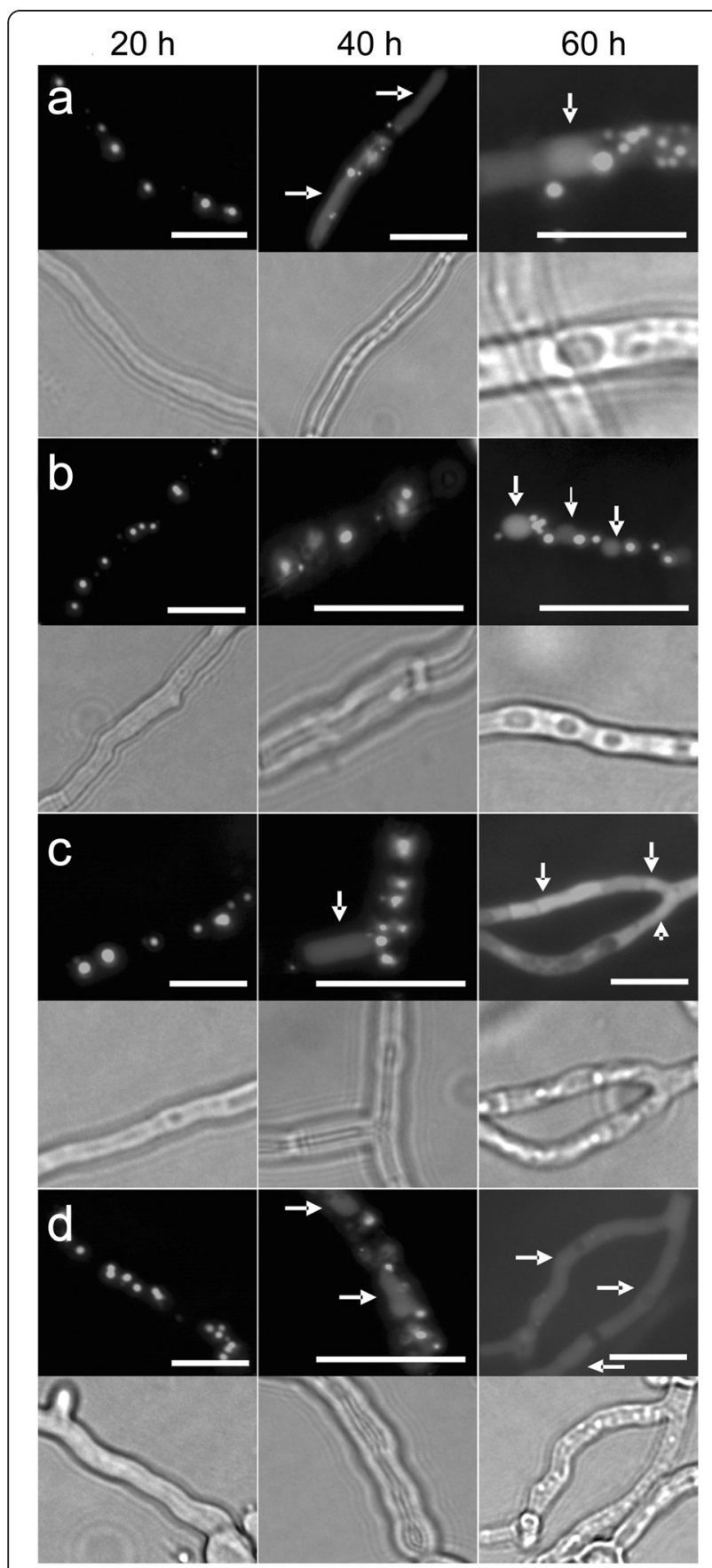

Fig. 2 Localisation of GFP-SKL in Ws54-1255 (GFP-SKL) treated with methylglyoxal and/or rapamycin. At the indicated times cultures grown on starvation pads supplemented with or without methylglyoxal and/ or rapamycin were analysed using fluorescence microscopy. Representative images are shown for each time point. a control; $\mathbf{b}+$ $0.05 \%(\mathrm{v} / \mathrm{v})$ methylglyoxal; c + $1 \mu \mathrm{M}$ rapamycin; $\mathbf{d}+0.05 \%(\mathrm{v} / \mathrm{v})$ methylglyoxal/+ $1 \mu \mathrm{M}$ rapamycin. Corresponding bright field areas are shown below each fluorescence channel image. White arrows: GFPSKL localised to vacuoles. Scale bars: $10 \mu \mathrm{m}$
Table 1 Analysis of autophagy in control (Ws54-1255 (GFP-SKL)) and $\Delta$ atg 1 (GFP-SKL) strains

\begin{tabular}{llll}
\hline & $20 \mathrm{~h}$ & $40 \mathrm{~h}$ & $60 \mathrm{~h}$ \\
\hline Control & - & + & ++ \\
$+\mathrm{MG}$ & - & - & + \\
$+\mathrm{R}$ & - & ++ & +++ \\
$+\mathrm{MG} /+\mathrm{R}$ & - & $(+)$ & + \\
$\Delta a t g 1$ & - & - & - \\
$+\mathrm{MG}$ & - & - & - \\
$+\mathrm{R}$ & - & - & - \\
$+\mathrm{MG} /+\mathrm{R}$ & - & - & - \\
\hline
\end{tabular}

For each data point around 100 hyphae were analysed by fluorescence microscopy. -: no peroxisome degradation observed, (+): weak peroxisome degradation (5-10\% of all hyphae), + : ca. $25 \%$ of hyphae display peroxisome degradation, ++: ca. $50 \%$ of hyphae display peroxisome degradation, +++: $90-100 \%$ of hyphae display peroxisome degradation.MG methylglyoxal $(0.05 \%[\mathrm{v} / \mathrm{v}]) . R$ rapamycin $(1 \mu \mathrm{M})$

the following observations are made (Fig. 2d): (i) at $20 \mathrm{~h}$, there is no apparent difference to the untreated control and exclusive methylglyoxal treatment, (ii) at $40 \mathrm{~h}$ a low level of peroxisome degradation is detected which is not seen in hyphae treated solely with methylglyoxal and (iii) at $60 \mathrm{~h}$ the tendency of GFP to localise to vacuoles is similar to samples treated with methylglyoxal alone. The phenotype at $40 \mathrm{~h}$ indicates that methylglyoxal cannot halt the induction of peroxisome degradation by rapamycin treatment.

As negative control for autophagy induction the $\Delta a t g 1$ (GFP-SKL) strain was utilized (Fig. 3) which is defective in both bulk (unselective) and selective autophagy [29]. This strain never shows a vacuolar localisation of GFP regardless whether there is methylglyoxal present in the growth medium or not (Fig. 3, Table 1). There are more peroxisomes present in $\Delta$ atg1 (GFP-SKL) compared to Ws54-1255 (GFP-SKL), an effect that has been previously reported [29]. These findings suggest that GFP-SKL is not transported to vacuoles by autophagy-independent processes.

Collectively the presented data suggest that methylglyoxal affects mycelium growth by delaying the entry into stationary phase.

\section{Effects of methylglyoxal on mitochondrial morphology}

Mitochondria display a strikingly dynamic morphology, ranging from small punctuate units to long thread-like structures. Their function depends on their morphology among other factors. 'Healthy' mitochondria, capable of synthesizing sufficient amounts of ATP, usually belong to the filamentous morphotype whereas spherical mitochondria are often an indication for cellular stress and death. It has been shown that mitochondrial morphology 


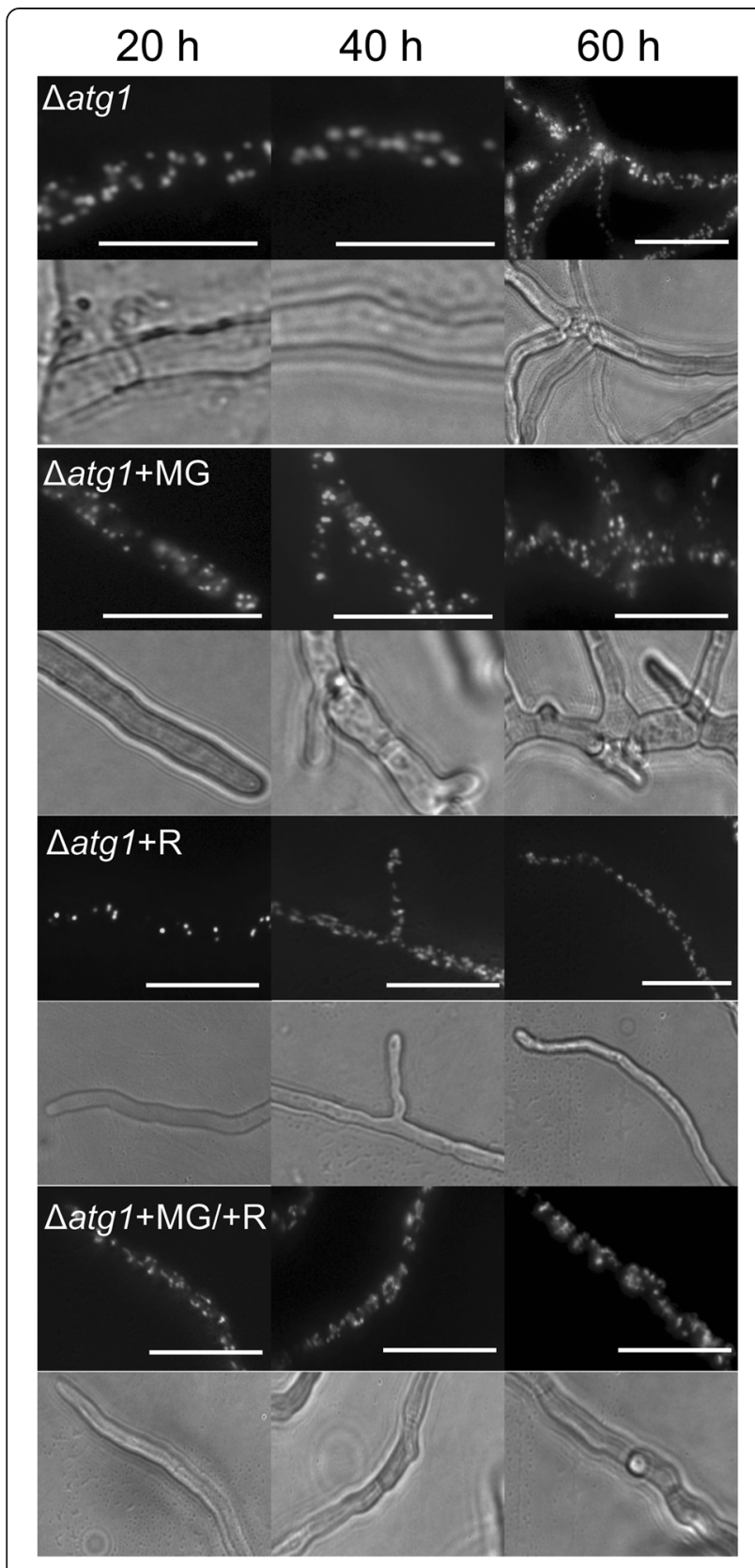

Fig. 3 Localisation of GFP-SKL in P. chrysogenum $\triangle$ atg1 (GFP-SKL) treated with methylglyoxal and/or rapamycin. At the indicated times cultures grown on starvation pads were analysed using fluorescence microscopy. Representative images are shown for each time point. Corresponding bright field areas are shown below each fluorescence channel image. + MG: $0.05 \%(v / v) ;+R: 1 \mu M$ rapamycin. Scale bars: $10 \mu \mathrm{m}$

can be used as a fungal viability marker [30, 31]. In order to address the question whether methylglyoxal and/or rapamycin affect viability of Ws54-1255 mitochondrial morphotypes were determined (Fig. 4, Table 2). Control cultures displayed a filamentous mitochondrial morphology up to $60 \mathrm{~h}$ of growth on the starvation pad, indicating that Ws54-1255 is capable to tolerate this pronounced starvation without the induction of cell death (Fig. 4a; Table 2). Cultures growing in the presence of $0.05 \%$ methylglyoxal exhibit a similar behaviour (Fig. 4b; Table 2). However, when cultures were subjected to $1 \mu \mathrm{M}$ rapamycin, hyphae contained mostly round/spherical mitochondria after 40 and $60 \mathrm{~h}$ of cultivation (Fig. 4c; Table 2). Although not demonstrated experimentally, it is suggested that this phenotype might be due to the induction of autophagy (mitophagy) as it is known that mitophagy is correlated with mitochondrial fragmentation [32]. Interestingly, when both methylglyoxal and rapamycin are applied, mitochondrial morphology is mostly filamentous after $40 \mathrm{~h}$ of incubation (Fig. 4d; Table 2). This finding suggests that methylglyoxal is able to counteract rapamycinmediated fragmentation of mitochondria. However, after $60 \mathrm{~h}$ of incubation hyphae contain mostly round (fragmented) mitochondria (Fig. 4d; Table 2).

\section{Response of $P$. chrysogenum to methylglyoxal at the protein level}

Methylglyoxal is known for its property to modify proteins. It is also known that some proteins are more susceptible for methylglyoxal-mediated glycation than others [5]. The aim of this part of the study was not to detect methylglyoxal-modified proteins but to elucidate the response of $P$. chrysogenum to methylglyoxal present in the growth medium (YGG + $0.2 \%$ [v/v] methylglyoxal). In this complete growth medium as opposed to growth on starvation pads (see above) P. chrysogenum was found to tolerate much higher methylglyoxal levels. After cultivation protein extracts from the untreated control and the methylglyoxal-treated sample were prepared and separated using isoelectric focusing and twodimensional SDS-PAGE. Staining with a highly sensitive Coomassie Blue variant revealed the separated proteins which were in the size range of 3-30 kDa (Fig. 5). A reference protein (Fig. 5, R) was selected that is present (i) in relatively high abundance, (ii) sufficiently isolated from other proteins to facilitate its punching out from the gel for characterisation and (iii) displays approximately the same staining intensity in the two gels (control and methylglyoxal). Three spots that are both isolated from other proteins to minimize the risk of ambiguous identification and present in higher abundance in the control gel compared to the gel containing the separated proteins from the methylglyoxal-treated culture were selected and punched out. Peptide mass fingerprinting revealed the identity of these proteins as follows: Reference protein - peptide from the $60 \mathrm{~S}$ ribosomal protein L5 (systematic identifier: Pc13g11570); \#1 - peptide from aldo-keto reductase (Pc22g04850, EC 1.1.1.2); \#2 - peptide from 5-methyl-tetrahydropteroyl-triglutamate-homocysteine S-methyltransferase (Pc22g18630, EC 2.1.1.14) and 


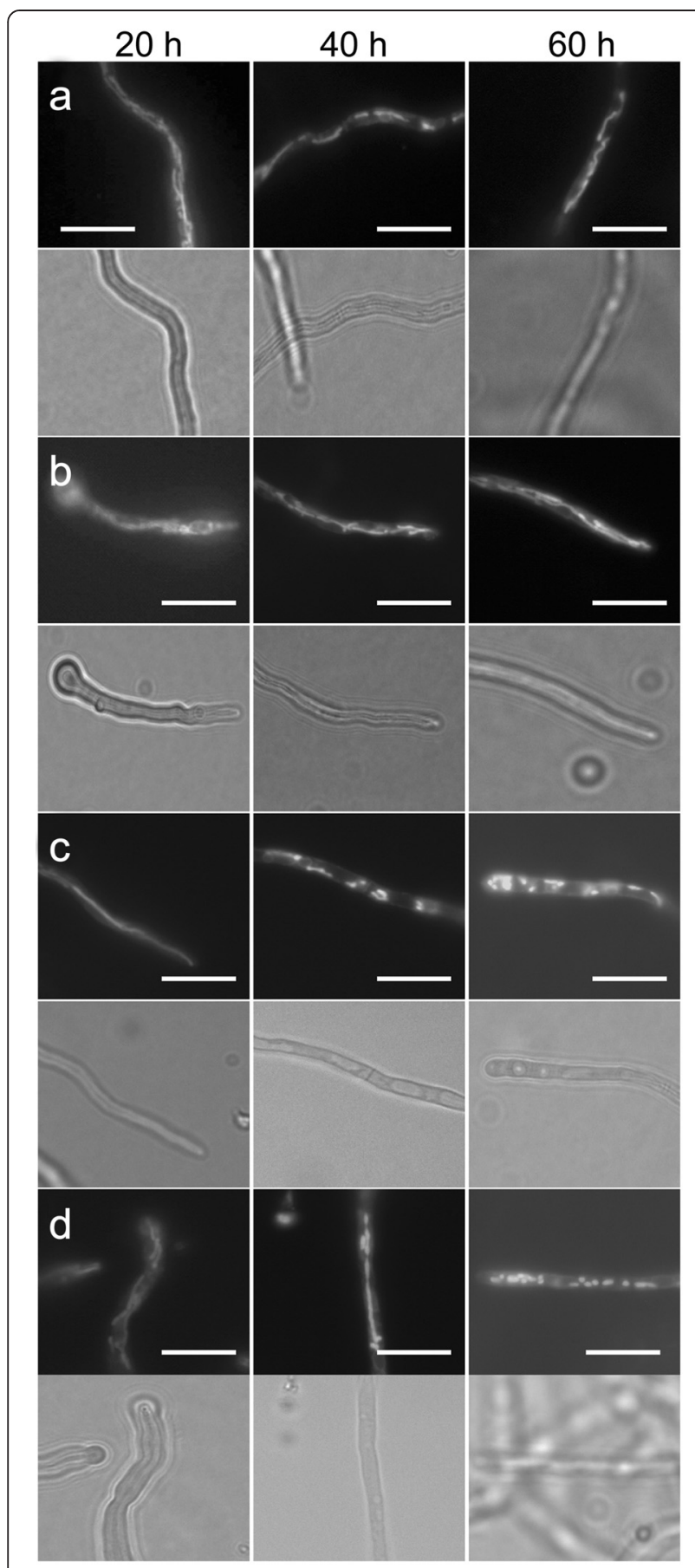

Fig. 4 Analysis of mitochondrial morphology in Ws54-1255 treated with methylglyoxal and/or rapamycin. At the indicated times cultures grown on starvation pads supplemented with or without methylglyoxal and/or rapamycin were overlaid with a Mitotracker Green FM solution and analysed using fluorescence microscopy. Representative images are shown for each time point. a control; b + $0.05 \%(\mathrm{v} / \mathrm{v})$ methylglyoxal; c + $1 \mu \mathrm{M}$ rapamycin; $\mathbf{d}+0.05 \%(\mathrm{v} / \mathrm{V})$ methylglyoxal/+ $1 \mu \mathrm{M}$ rapamycin. Corresponding bright field areas are shown below each fluorescence channel image. Scale bars: $10 \mu \mathrm{m}$
Table 2 Mitochondrial morphotypes in Ws54-1255

\begin{tabular}{llll}
\hline & $20 h$ & $40 h$ & $60 h$ \\
\hline Control & fil & fil & fil \\
$+M G$ & fil & fil & fil \\
$+R$ & fil & rnd & rnd \\
$+M G /+R$ & fil & fil & rnd \\
\hline
\end{tabular}

fil mostly filamentous mitochondria. rnd mostly round, spherical mitochondria. MG methylglyoxal $(0.05 \%[\mathrm{v} / \mathrm{v}]) . R$ rapamycin $(1 \mu \mathrm{M})$

\#3 - peptide from NAD-dependent formate dehydrogenase (Pc12g04310, EC 1.2.1.2). Two of the identified peptides (\#1 and \#3) belong to proteins that may play a direct role in $\alpha$-oxoaldehyde metabolism while \#2 is involved in homocysteine/methionine metabolism (see Discussion).

\section{Discussion}

The results shown here suggest that methylglyoxal reduces growth of $P$. chrysogenum so that the onset of the stationary phase is delayed. Degradation of peroxisomes by autophagy in the presence or absence of methylglyoxal was utilized to monitor entry into stationary phase. It is widely known that methylglyoxal is capable to readily react with proteins. Are peroxisomal proteins subjected to glycation? In Arabidopsis thaliana it was shown that a glyoxalase I homolog, GLX1, is present in peroxisomes [33]. This result hints that there is a need for the removal of methylglyoxal at least in plant peroxisomes (although a GLX2 activity has so far not been demonstrated in plant peroxisomes). It should be kept in mind that the glyoxalase system is not the only line of defense against the toxic effects of $\alpha$-oxoaldehydes. In addition to the aldehyde reductases and dehydrogenases described in the introduction there are also more indirect mechanisms to prevent the formation of methylglyoxal. In rice it was shown that an increase in methylglyoxal levels correlated with an elevated formation of the glycolysis enzyme triose phosphate isomerase [34]. This reaction results in reduced formation of DHAP which is giving rise to methylglyoxal by spontaneous phosphate elimination (see introduction). So far it is not known if a similar mechanism exists in other organisms but the possibility is certainly intriguing.

Interestingly, methylglyoxal was found to stimulate autophagy in bovine aortic endothelial cells [35]. The authors found increased levels of the autophagy marker proteins Beclin-1 and LC3B-II after methylglyoxal treatment. Furthermore, autophagy flux, as indicated by the appearance of GFP-LC3 dots, was significantly increased in the methylglyoxal-treated cells. It is not known whether other mammalian cell types respond to methylglyoxal in a comparable manner by up-regulating the molecular autophagy machinery.

No candidates of the molecular machinery regulating autophagy were identified in the IEF/2D-SDS-PAGE 

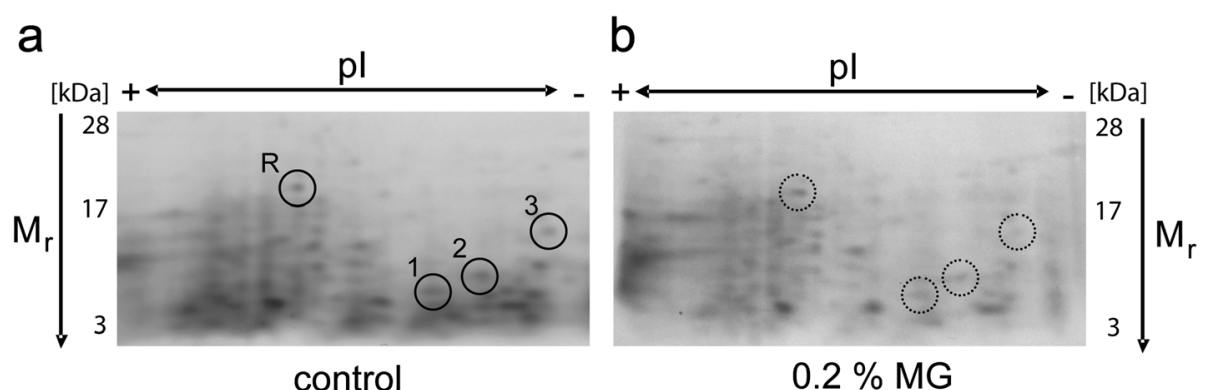

Fig. 5 Analysis of changes of protein abundance by methylglyoxal treatment in Ws54-1255 by isoelectric focusing/2D-SDS-PAGE/peptide mass fingerprinting. a Separated proteins of an untreated Ws54-1255 culture grown in YGG medium for 2.5 days at $25^{\circ} \mathrm{C}$ visualized by Coomassie Blue staining. Proteins with different abundance compared to the culture grown in the presence of $0.2 \%(\mathrm{v} / \mathrm{V})$ methylglyoxal are marked by black circles. The identity of selected peptide spots was determined by peptide mass fingerprinting: R-peptide belonging to $60 \mathrm{~S}$ ribosomal protein $\mathrm{L} 5$ (Pc13g11570); 1-peptide belonging to aldo-keto reductase (Pc22g04850); 2-peptide belonging to 5-methyl-tetrahydropteroyl-triglutamatehomocysteine S-methyltransferase (Pc22g18630); 3-peptide belonging to NAD-dependent formate dehydrogenase (Pc12g04310). b Separated proteins of a Ws54-1255 culture grown in YGG medium for 2.5 days at $25^{\circ} \mathrm{C}$ in the presence of $0.2 \%(\mathrm{v} / \mathrm{V})$ methylglyoxal visualized by Coomassie Blue staining. Regions corresponding to the gel shown in panel a are marked with dotted circles. pl: isoelectric point, MG: methylglyoxal, $\mathrm{M}_{\mathrm{r}}$ : relative molecular mass

experiment. However, peptides from three proteins were revealed that are present in reduced abundance when the fungus is grown in medium containing methylglyoxal. Aldo-keto reductase (Pc22g04850) and the NAD-dependent formate dehydrogenase (Pc12g04310) have so far not been characterized in detail in P. chrysogenum. It is certainly possible that both proteins are involved in methylglyoxal metabolism/detoxification. NADdependent formate dehydrogenase from the methylotrophic yeast Candida boidinii was shown to accept methylglyoxal as a substrate in addition to formaldehyde [36]. Furthermore, AKR4C14, a rice aldo-keto reductase, from Thai Jasmine rice is capable to metabolise methylglyoxal in addition to sugars and the aldehydes glutaraldehyde and trans-2-hexenal [37]. Therefore Pc22g04850 and Pc12g04310 might constitute components of a cellular defense system that supports the glyoxalase system in keeping cellular levels of methylglyoxal low. The observation that these proteins are actually present in decreased abundance when $P$. chrysogenum is subjected to methylglyoxal could be explained by a 'methylglyoxal overload effect': the proteins are extensively modified by their reaction with methylglyoxal so that they become damaged and are subsequently degraded. Due to the rather high methylglyoxal concentration applied in the experiment the cell fails to maintain a sufficient supply of these enzymes. This would make the corresponding genes prime candidates for their overexpression to further improve the defense capacity of $P$. chrysogenum against methylglyoxal and related $\alpha$-oxoaldehydes with possibly beneficial effects on biosynthetic productivity. The protein analysis also identified 5-methyl-tetrahydropteroyl-triglutamate-homocysteine S-methyltransferase (Pc22g18630) as a protein which is present in lowered amounts when methylglyoxal is added to the growth medium of $P$. chrysogenum. The enzyme catalyses the final step in methionine biosynthesis (5methyltetrahydropteroyltri-l-glutamate + l-homocysteine $\rightarrow$ tetrahydropteroyltri-l-glutamate $+\mathrm{l}$-methionine) [38]. This reaction is also important for keeping cellular levels of homocysteine low. Homocysteine has several deleterious properties if its cellular levels are elevated, among them apoptosis induction, increased formation of toxic reactive oxygen species and interference with cellular signalling (e. g., due to DNA and RNA methylation, etc. [39]). Therefore it is possible that cellular stress caused by increased methylglyoxal levels and a decrease of 5-methyl-tetrahydropteroyl-triglutamate-homocysteine Smethyltransferase results in the amplification of deleterious signals which could lead to severe cellular defects. For example, the observed inhibitory effect of methylglyoxal on peroxisome degradation could also be based on its indirect effects on other metabolic pathways (i. e., homocysteine metabolism).

\section{Conclusions}

Methylglyoxal reduces growth of $P$. chrysogenum cultivated on starvation pads so that the onset of stationary phase (as monitored by the degradation of GFP-SKL labelled peroxisomes via autophagy) is delayed. Mitochondrial morphology is not compromised by methylglyoxal: the thread-like morphology is not changed and mitochondrial fragmentation is not observed. Three proteins were identified that are present in lower abundance when methylglyoxal is added to a liquid culture of $P$. chrysogenum: \#1Aldo-keto reductase (Pc22g04850); \#2-5-methyl-tetrahydropteroyl-triglutamate-homocysteine S-methyltransferase (Pc22g18630) and \#3-NAD-dependent formate dehydrogenase (Pc12g04310). 


\section{Methods}

\section{Strains and cultivation conditions}

Penicillium chrysogenum Wisconsin(Ws)54-1255 (GFPSKL) [40] was used for growth experiments and for the localisation of green fluorescent protein targeted to peroxisomes (GFP-SKL). Stocks grown on rice were stored at room temperature (RT) in the dark. For initiating growth from stocks and for the preparation of fresh stocks 2-3 rice grains were placed on YGG agar (10 g/l $\mathrm{KCl}, 20 \mathrm{~g} / \mathrm{l}$ glucose, $10 \mathrm{~g} / \mathrm{l}$ yeast nitrogen base, $5 \mathrm{~g} / \mathrm{l}$ $\mathrm{K}_{2} \mathrm{HPO}_{4}, 20 \mathrm{~g} / \mathrm{l}$ yeast extract, $20 \mathrm{~g} / \mathrm{l}$ agar-agar) and incubated for 4 to 5 days at RT. For cultivation in liquid medium 15-20 rice grains covered with mycelium were inoculated in $50 \mathrm{ml}$ YGG medium in a plastic tube for $1 \mathrm{~h}$ with occasional mixing. Afterwards the culture (without the rice grains) was transferred to a $250 \mathrm{ml}$ Erlenmeyer piston. Cultures were incubated for 2 to 3 days at $25{ }^{\circ} \mathrm{C}$ at $180 \mathrm{rpm}$ in an Ecotron shaker (Infors $\mathrm{GmbH}$, Einsbach, Germany). As an autophagy negative control for the assessment of degradation of peroxisomes the $P$. chrysogenum strain $\Delta a \operatorname{tg} 1$ (GFP-SKL) [29] was used in which all autophagy pathways are blocked. Methylglyoxal (cat. no. M0252, Sigma-Aldrich, Taufkirchen, Germany) was added to the media as specified in the corresponding subsections.

\section{Microscopy}

\section{Cultivation of $P$. chrysogenum for microscopy}

For cultivating the Ws54-1255 (GFP-SKL) strain 2-3 rice grains covered with sporulating mycelium ('green rice') were incubated in $500 \mu \mathrm{l}$ YGG medium in a $1.5 \mathrm{ml}$ microcentrifuge tube for $1 \mathrm{~d}$ at RT after vortexing the tube for $30 \mathrm{~s}$. From this spore suspension a 1/50 dilution in sterile tap water was prepared. $5 \mu \mathrm{l}$ of this diluted suspension were pipetted onto an agarose 'starvation' pad $(1 \%(\mathrm{w} / \mathrm{v})$ agarose in sterile tap water containing no additives (control) or $0.05 \%$ (v/v) methylglyoxal and/or $1 \mu \mathrm{M}$ rapamycin) that was mounted on an object carrier with a central cavity [25]. Samples were incubated at RT for up to $60 \mathrm{~h}$ in a wet chamber to prevent desiccation.

For cultivation of the $\Delta a t g 1$ (GFP-SKL) strain that has a reduced ability to sporulate [29] $5 \mu$ l of an undiluted spore suspension was pipetted onto an YGG agar plate and incubated for $5 \mathrm{~d}$ at RT. This resulted in the growth of a mycelium colony. Small pieces of mycelium were transferred with a sterile toothpick into a $1.5 \mathrm{ml}$ microcentrifuge tube containing $400 \mu$ l sterile tap water and approx. $100 \mathrm{mg}$ glass beads $(0.25 \mathrm{~mm}-0.5 \mathrm{~mm}$ diameter, Carl Roth $\mathrm{GmbH}$, Karlsruhe, Germany). This suspension was vortexed for $2 \mathrm{~min}$. The treatment resulted in fragmentation of the mycelium. $5 \mu \mathrm{l}$ of the undiluted suspension was pipetted onto a starvation pad and incubated as described above.

\section{Microscopy analysis}

Samples were analysed with an Axio Imager.M2 fluorescence microscope equipped with an AxioCam Mrc5 digital camera (Carl Zeiss GmbH, Goettingen, Germany). Images documenting mycelium growth (bright-field microscopy) were processed to improve visibility of hyphae using Photoshop 5.0 (Adobe, San José, CA) as follows: (i) inverting the picture (pos $>$ neg), (ii) adjustment of brightness/contrast until hyphae were well resolved. The same settings were applied to all images to allow comparison.

For studying mitochondrial morphology mycelia grown on starvation pads were carefully overlaid with $10 \mu \mathrm{M}$ Mitotracker Green FM (cat. no. M-7514, Fisher Scientific, Schwerte, Germany) solution for $10 \mathrm{~min}$ before analysis by fluorescence microscopy.

\section{Protein extraction}

P. chrysogenum cultures were grown for $2.5 \mathrm{~d}$ at $25{ }^{\circ} \mathrm{C}$ (180 rpm) in YGG medium (either in the absence or presence of $0.2 \%[\mathrm{v} / \mathrm{v}]$ methylglyoxal). After filtration the mycelium was briefly washed by rinsing it with sterile water. A flat paste of mycelium was wrapped in aluminium foil and stored overnight in a freezer at $-25{ }^{\circ} \mathrm{C}$. The next day the frozen mycelium was quickly ground to a fine powder in a chilled mortar containing cold glass beads $(0.25 \mathrm{~mm}-0.5 \mathrm{~mm}$ diameter, Carl Roth $\mathrm{GmbH}$, Karlsruhe, Germany) to prevent thawing. The ground powder was stirred in cold isoelectric focusing buffer $(8 \mathrm{M}$ urea, $2 \%[\mathrm{w} / \mathrm{v}$ ] CHAPS, $0.5 \%$ [v/v] ampholyte solution pH 3-10 [Fisher Scientific, Schwerte, Germany], 1/1000 protease inhibitor cocktail [cat. no. P8215, Sigma-Aldrich, Taufkirchen, Germany]) at $4{ }^{\circ} \mathrm{C}$ for $1 \mathrm{~h}$. After centrifugation (10 $\mathrm{min}, 6000 \mathrm{x} \mathrm{g}, 4{ }^{\circ} \mathrm{C}$ ) the supernatant was carefully removed and an aliquot was analysed for its protein concentration. To the rest of the extract bromophenolblue (final concentration: $0.002 \%[\mathrm{w} / \mathrm{v}]$ ) and 1,4-dithio-DLthreitol (DTT) (final concentration: $20 \mathrm{mM}$ ) were added. Care was taken that protein extracts were always thawed on ice and subsequently kept chilled when removed from the freezer.

\section{Protein concentration determination}

Preparations were assayed for the protein concentration by using the 'Quantipro BCA assay kit' (Sigma Aldrich, Taufkirchen, Germany) according to the manufacturer's instructions. An aliquot of the extract was diluted 1/10 in isoelectric focusing buffer (without bromophenolblue and DTT due to the interference of these compounds with the BCA assay). Samples were incubated for $1 \mathrm{~h}$ at $60{ }^{\circ} \mathrm{C}$ before absorbance was read at $562 \mathrm{~nm}$. Values were compared to a reference curve prepared with different dilutions of a bovine serum albumin solution (supplied with the kit). 


\section{Isoelectric focusing/2D-SDS-PAGE}

For isoelectric focusing and 2D-SDS-PAGE the ZOOM system (Fisher Scientific, Schwerte, Germany) was used according to the manufacturer's instructions. Isoelectric focusing buffer (containing $500 \mu \mathrm{g}$ P. chrysogenum protein extract) was loaded onto $\mathrm{pH}$ 3-10 NL (non-linear) IEF strips (Fisher Scientific, Schwerte, Germany) and incubated at $4{ }^{\circ} \mathrm{C}$ overnight for rehydration of the polyacrylamide gel on the strips. IEF was performed with the following voltage settings: $1 \mathrm{~h} 100 \mathrm{~V}, 2 \mathrm{~h} 200 \mathrm{~V}, 3 \mathrm{~h}$ $500 \mathrm{~V}$. After the run strips were incubated for $15 \mathrm{~min}$ in $1 \times$ NuPAGE LDS sample buffer (Fisher Scientific, Schwerte, Germany) containing 1/10 NuPAGE sample reducing agent (Fisher Scientific, Schwerte, Germany) on a shaker at RT. A similar incubation step for $15 \mathrm{~min}$ in $1 \mathrm{x}$ NuPAGE LDS sample buffer containing $125 \mathrm{mM}$ iodoacetamide followed. Strips were then slid onto ZOOM gels (4-12\% polyacrylamide gradient gels, Fisher Scientific, Schwerte, Germany) and immobilised with a prewarmed solution containing $0.5 \%(\mathrm{w} / \mathrm{v})$ agarose in $1 \mathrm{x}$ NuPAGE MES running buffer (Fisher Scientific, Schwerte, Germany). 2D-SDS-PAGE was performed for $45 \mathrm{~min}$ at $200 \mathrm{~V}$ in $1 \mathrm{x}$ NuPAGE MES running buffer.

\section{Staining and documentation of polyacrylamide gels}

For the detection of separated proteins in polyacrylamide gels the 'Colloidal Blue staining kit' (Fisher Scientific, Schwerte, Germany) was used according to the manufacturer's instructions with the following alterations: gels were incubated overnight in staining solution and destained in pure water (changed every two hours) for at least $6 \mathrm{~h}$. For documentation purposes gels were placed on an illumination box (LP200, Doerr, Neu-Ulm, Germany) equipped with a red light filter and photographed using a digital camera.

\section{Protein identification}

Proteins spots of interest were punched out from stained SDS-PAGE gels by using cut pipette tips and analysed by mass spectrometry (MALDI-TOF peptide mass fingerprinting) by Alphalyse A/S, Odense, Denmark. For protein identification the NCBInr database was accessed using Mascot version 2.4. Peptide ions were considered to be positively identified when their Mascot score was above the $95 \%$ confidence level (i. e., $>=90$ for the NCBInr database).

\section{Abbreviations}

AGE: Advanced glycation end-product; DHAP: Dihydroxyacetone phosphate; DTT: 1,4-dithio-DL-threitol; GFP: Green fluorescent protein; GLO1: Glyoxalase I; GLO2: Glyoxalase II; IAT: Isopenicillin-N acyltransferase; MG: Methylglyoxal; YGG: Yeast glucose growth.

\section{Competing interests}

The author declares that he has no competing interests.

\section{Author's contributions}

CQS designed the research project, performed the experiments, analysed the data and wrote the manuscript.

\section{Acknowledgements}

CQS receives a fellowship from the LOEWE Excellence Cluster for Integrative Fungal Research (IPF). I would like to thank Ida J. van der Klei for the P. chrysogenum strains used in this study and Andreas S. Reichert for the gift of rapamycin.

Received: 25 March 2015 Accepted: 26 June 2015

Published online: 10 July 2015

\section{References}

1. Rabbani N, Thornalley PJ. Methylglyoxal, glyoxalase 1 and the dicarbonyl proteome. Amino Acids. 2012;42:1133-42.

2. Phillips SA, Thornalley PJ. The formation of methylglyoxal from triose phosphates. Investigation using a specific assay for methylglyoxal. Eur J Biochem. 1993;212:101-5.

3. Lyles GA, Chalmers J. The metabolism of aminoacetone to methylglyoxal by semicarbazide-sensitive amine oxidase in human umbilical artery. Biochem Pharmacol. 1992;43:1409-14.

4. Esterbauer H, Cheeseman KH, Dianzani MU, Poli G, Slater TF. Separation and characterization of the aldehydic products of lipid peroxidation stimulated by ADP-Fe ${ }^{2+}$ in rat liver microsomes. Biochem J. 1982;208:129-40.

5. Gomes RA, Vicente MH, Silva MS, Graca G, Coelho AV, Ferreira AE, et al. Yeast protein glycation in vivo by methylglyoxal. Molecular modification of glycolytic enzymes and heat shock proteins. FEBS J. 2006;273:5273-87.

6. Ahmed N, Thornalley PJ, Dawczynski J, Franke S, Strobel J, Stein G, et al. Methylglyoxal-derived hydroimidazolone advanced glycation end-products of human lens proteins. Invest Ophthalmol Vis Sci. 2003:44:5287-92.

7. Sousa SM, Gomes RA, Ferreira AE, Ponces FA, Cordeiro C. The glyoxalase pathway: the first hundred years... and beyond. Biochem J. 2013;453:1-15.

8. Dobler D, Ahmed N, Song L, Eboigbodin KE, Thornalley PJ. Increased dicarbonyl metabolism in endothelial cells in hyperglycemia induces anoikis and impairs angiogenesis by RGD and GFOGER motif modification. Diabetes. 2006;55:1961-9.

9. Gao Y, Wang Y. Site-selective modifications of arginine residues in human hemoglobin induced by methylglyoxal. Biochemistry. 2006;45:15654-60.

10. Ahmed N, Babaei-Jadidi R, Howell SK, Beisswenger PJ, Thornalley PJ. Degradation products of proteins damaged by glycation, oxidation and nitration in clinical type 1 diabetes. Diabetologia. 2005;48:1590-603.

11. Gomes RA, Oliveira LM, Silva M, Ascenso C, Quintas A, Costa G, et al. Protein glycation in vivo: functional and structural effects on yeast enolase. Biochem J. 2008:416:317-26.

12. Rabbani N, Xue M, Thornalley PJ. Activity, regulation, copy number and function in the glyoxalase system. Biochem Soc Trans. 2014;42:419-24.

13. Morcos M, Du X, Pfisterer F, Hutter H, Sayed AA, Thornalley P, et al. Glyoxalase-1 prevents mitochondrial protein modification and enhances lifespan in Caenorhabditis elegans. Aging Cell. 2008;7:260-9.

14. Lee JY, Song J, Kwon K, Jang S, Kim C, Baek K, et al. Human DJ-1 and its homologs are novel glyoxalases. Hum Mol Genet. 2012;21:3215-25.

15. Bonifati V, Rizzu P, van Baren MJ, Schaap O, Breedveld GJ, Krieger E, et al. Mutations in the DJ-1 gene associated with autosomal recessive early-onset parkinsonism. Science. 2003;299:256-9.

16. Irrcher I, Aleyasin H, Seifert EL, Hewitt SJ, Chhabra S, Phillips M, et al. Loss of the Parkinson's disease-linked gene DJ-1 perturbs mitochondrial dynamics. Hum Mol Genet. 2010;19:3734-46.

17. Giaime E, Yamaguchi H, Gautier CA, Kitada T, Shen J. Loss of DJ-1 does not affect mitochondrial respiration but increases ROS production and mitochondrial permeability transition pore opening. PLoS One. 2012;7:e40501

18. Toyoda Y, Erkut C, Pan-Montojo F, Boland S, Stewart MP, Müller DJ, et al. Products of the Parkinson's disease-related glyoxalase DJ-1, D-lactate and glycolate, support mitochondrial membrane potential and neuronal survival. Biol Open. 2014;3(8):777-84. BIO20149399.

19. Chen CN, Porubleva L, Shearer G, Svrakic M, Holden LG, Dover UL, et al Associating protein activities with their genes: rapid identification of a gene encoding a methylglyoxal reductase in the yeast Saccharomyces cerevisiae. Yeast. 2003;20:545-54. 
20. Lyon RC, Li D, McGarvie G, Ellis EM. Aldo-keto reductases mediate constitutive and inducible protection against aldehyde toxicity in human neuroblastoma SH-SY5Y cells. Neurochem Int. 2013;62:113-21.

21. Vander Jagt $\mathrm{DL}$, Hunsaker LA. Methylglyoxal metabolism and diabetic complications: roles of aldose reductase, glyoxalase-l, betaine aldehyde dehydrogenase and 2-oxoaldehyde dehydrogenase. Chem Biol Interact. 2003;143-144:341-51.

22. Scheckhuber CQ, Mack SJ, Strobel I, Ricciardi F, Gispert S, Osiewacz HD Modulation of the glyoxalase system in the aging model Podospora anserina: effects on growth and lifespan. Aging (Albany NY). 2010;2:969-80.

23. Scheckhuber $C Q$, Veenhuis $M$, van der Klei I. Improving penicillin biosynthesis in Penicillium chrysogenum by glyoxalase overproduction. Metab Eng. 2013;18:36-43.

24. Lutz MV, Bovenberg RA, van der Klei I, Veenhuis M. Synthesis of Penicillium chrysogenum acetyl-CoA:isopenicillin N acyltransferase in Hansenula polymorpha: first step towards the introduction of a new metabolic pathway. FEMS Yeast Res. 2005:5:1063-7.

25. Scheckhuber CQ. Analysis of autophagy in Penicillium chrysogenum by using starvation pads in combination with fluorescence microscopy. J Vis Exp. 2015;96:52577.

26. Heitman J, Movva NR, Hall MN. Targets for cell cycle arrest by the immunosuppressant rapamycin in yeast. Science. 1991;253:905-9.

27. Jung $\mathrm{CH}, \mathrm{Ro} \mathrm{SH}, \mathrm{Cao} J$, Otto NM, Kim DH. mTOR regulation of autophagy. FEBS Lett. 2010:584:1287-95.

28. Cubitt AB, Heim R, Adams SR, Boyd AE, Gross LA, Tsien RY. Understanding, improving and using green fluorescent proteins. Trends Biochem Sci. 1995;20:448-55.

29. Bartoszewska M, Kiel JA, Bovenberg RA, Veenhuis M, van der Klei I. Autophagy deficiency promotes beta-lactam production in Penicillium chrysogenum. Appl Environ Microbiol. 2011;77:1413-22.

30. Scheckhuber CQ, Erjavec N, Tinazli A, Hamann A, Nystrom T, Osiewacz HD. Reducing mitochondrial fission results in increased life span and fitness of two fungal ageing models. Nat Cell Biol. 2007;9:99-105.

31. Kato A, Kurashima K, Chae M, Sawada S, Hatakeyama S, Tanaka S, et al. Deletion of a novel F-box protein, MUS-10, in Neurospora crassa leads to altered mitochondrial morphology, instability of mtDNA and senescence. Genetics. 2010;185:1257-69.

32. Mao K, Wang K, Liu X, Klionsky DJ. The scaffold protein Atg11 recruits fission machinery to drive selective mitochondria degradation by autophagy. Dev Cell. 2013;26:9-18.

33. Quan S, Switzenberg R, Reumann S, Hu J. In vivo subcellular targeting analysis validates a novel peroxisome targeting signal type 2 and the peroxisomal localization of two proteins with putative functions in defense in Arabidopsis. Plant Signal Behav. 2010;5:151-3.

34. Sharma S, Mustafiz A, Singla-Pareek SL, Shankar SP, Sopory SK Characterization of stress and methylglyoxal inducible triose phosphate isomerase (OscTPI) from rice. Plant Signal Behav. 2012;7:1337-45.

35. Liu H, Yu S, Zhang H, Xu J. Angiogenesis impairment in diabetes: role of methylglyoxal-induced receptor for advanced glycation endproducts, autophagy and vascular endothelial growth factor receptor 2. PLoS One 2012;7:e46720

36. Schüte H, Flossdorf J, Sahm H, Kula MR. Purification and properties of formaldehyde dehydrogenase and formate dehydrogenase from Candida boidinii. Eur J Biochem. 1976;62:151-60.

37. Narawongsanont R, Kabinpong S, Auiyawong B, Tantitadapitak C. Cloning and characterization of AKR4C14, a rice aldo-keto reductase, from Thai Jasmine rice. Protein J. 2012:31:35-42

38. Whitfield CD, Steers Jr EJ, Weissbach H. Purification and properties of 5-methyltetrahydropteroyltriglutamate-homocysteine transmethylase. J Biol Chem. 1970;245:390-401.

39. Boldyrev AA. Molecular mechanisms of homocysteine toxicity. Biochemistry (Mosc). 2009:74:589-98

40. Meijer WH, Gidijala L, Fekken S, Kiel JA, van den Berg MA, Lascaris R, et al. Peroxisomes are required for efficient penicillin biosynthesis in Penicillium chrysogenum. Appl Environ Microbiol. 2010;76:5702-9.

\section{Submit your next manuscript to BioMed Central and take full advantage of:}

- Convenient online submission

- Thorough peer review

- No space constraints or color figure charges

- Immediate publication on acceptance

- Inclusion in PubMed, CAS, Scopus and Google Scholar

- Research which is freely available for redistribution

Submit your manuscript at www.biomedcentral.com/submit 